Musées, Patrimoine et Culture scientifiques et techniques

$191 \mid 2020$

septembre-octobre 2020

\title{
Archéocapsule la recherche archéologique exposée en itinérance
}

Alessia Bonannini

\section{OpenEdition}

\section{Journals}

Édition électronique

URL : https://journals.openedition.org/ocim/4097

DOI : 10.4000/ocim.4097

ISSN : 2108-646X

Éditeur

OCIM

Édition imprimée

Date de publication : 1 septembre 2020

Pagination : 8-15

ISSN : 0994-1908

Référence électronique

Alessia Bonannini, « Archéocapsule la recherche archéologique exposée en itinérance », La Lettre de I'OCIM [En ligne], 191 | 2020, mis en ligne le 01 septembre 2021, consulté le 12 décembre 2021. URL http://journals.openedition.org/ocim/4097 ; DOI : https://doi.org/10.4000/ocim.4097

Ce document a été généré automatiquement le 12 décembre 2021.

Tous droits réservés 


\title{
Archéocapsule la recherche archéologique exposée en itinérance
}

\author{
Alessia Bonannini
}

L'Archéocapsule Archéologie de l'esclavage au musée de l'Homme, 2019.

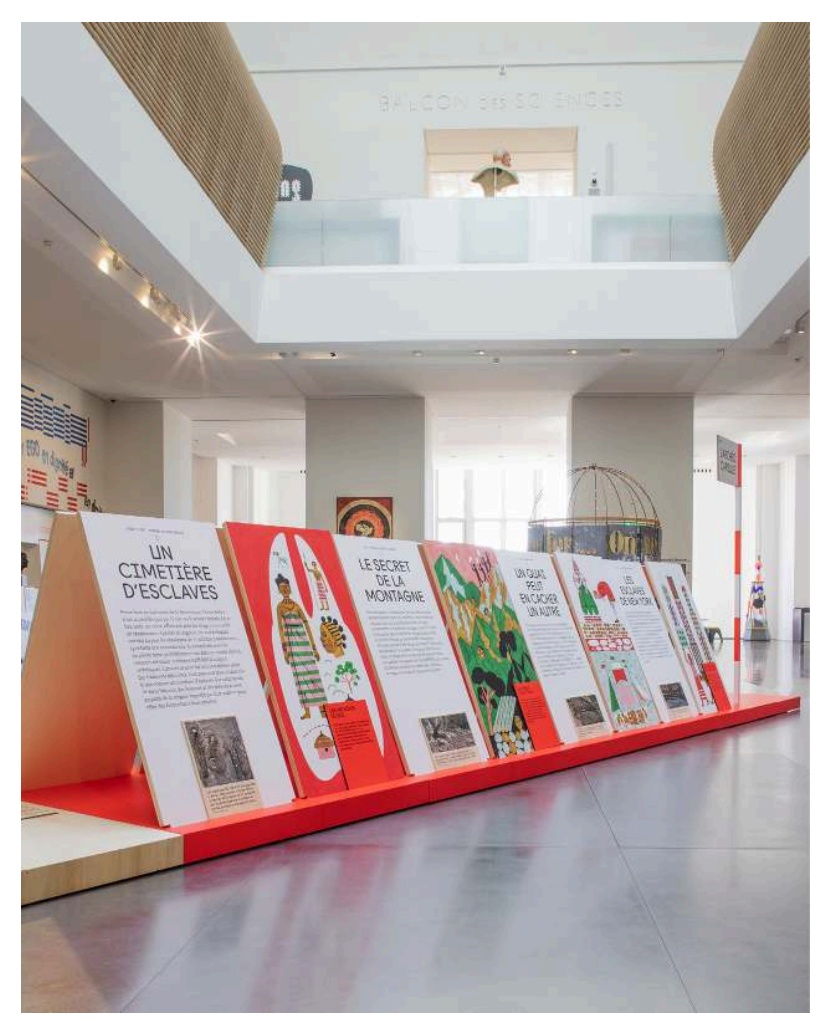

(c) J. Lelièvre/Inrap 


\section{L'itinérance ou le défi de la greffe}

1 La pratique des expositions itinérantes, qui se généralise sous la double impulsion de considérations culturelles et économiques, peut mettre en jeu, et parfois même en danger, le sens et l'identité des institutions. Comme une greffe à la réussite incertaine, elle pose la question de l'adaptation d'une production - dans son contenu et dans sa forme - à un lieu d'accueil et à un public. Si les formules «clés en main » offrent des avantages certains, elles interrogent la pertinence et le respect des missions statutaires des institutions accueillantes, que l'on voit parfois sacrifiées ou artificiellement élargies. L'entrée des sponsors dans l'arène complexifie d'autant plus la démarche, poussant parfois jusqu'à une forme de renoncement identitaire de l'institution d'accueil.

2 Des propositions plus souples, avec des contenus adaptables, pouvant être modifiés et prendre un élan vital du fait de leur appropriation possible, sont également développées, ce qui engage une posture différente des partenaires, dans une logique de co-production, au sens culturel du mot. Un dialogue s'installe entre les parties et génère un modèle culturel vertueux, bien que plus complexe, plus consommateur de temps et à l'équilibre financier plus délicat.

3 Le nouveau dispositif d'expositions itinérantes développé par l'Institut national de recherches archéologiques préventives (Inrap) s'insère dans cette mouvance. Il a fallu cependant considérer très précisément les objectifs et les contraintes spécifiques à l'institution, pour arriver à définir le modèle qui «fait sens » tant pour l'Institut, que pour le public.

\section{Assumer une posture}

Produire des expositions itinérantes en maitrise d'ouvrage propre est une première pour l'Inrap. Une politique de partenariats d'envergure avec des musées nationaux et régionaux y est développée depuis la création en 2005 de la direction du Développement culturel et de la Communication, débouchant parfois sur des grandes co-productions. Tel a été le cas par exemple de l'exposition Gaulois, une expo renversante (2014) et Quoi de neuf au Moyen-Âge (2016) avec la Cité des sciences et de l'industrie, Néandertal (2018) avec le musée de l'Homme, ou Tromelin, l'île aux esclaves oubliés (2016) avec le musée d'Histoire de Nantes et le Gran'1. Par ailleurs, produire des expositions légères - sur panneaux ou supports de type roll-up - est une pratique solidement installée.

5 Ces deux axes de développement des expositions sont liés à la nature même de l'Institut et du contexte préventif. Doté par le Code du patrimoine de missions de diffusion des connaissances archéologique auprès des publics, l'Inrap ne possède pas un lieu propre d'exposition (en dehors bien sûr du terrain de fouille lui-même). Ainsi, pour ce qui relève du développement des actions de monstration, l'établissement cumule le plus grand avantage (la primeur des vestiges et des données) et la plus grande difficulté (le caractère éphémère de l'intervention archéologique). Celle-ci se déroule pendant un laps de temps fixé en amont et souvent incompressible, dans des conditions d'accueil des publics complexes, sans qu'une interprétation soit immédiatement livrable, et vouée à la disparition ou plutôt à la «sauvegarde par l'étude $»^{2}$ dans la majorité des cas. 
Le mobilier issu des fouilles est quant à lui versé à l'État et finalement exposé dans les musées après une période d'étude et de recherche.

Dans ce contexte, l'investissement d'un rôle de maîtrise d'ouvrage avec des productions d'expositions propres répond d'abord à la volonté d'affirmer une présence constante et pérenne de l'Inrap dans l'environnement culturel, de manière indépendante du calendrier et des missions des partenaires. Il répond aussi à l'urgence de la communication autour des fouilles et des objets fouillés eux-mêmes. Tout en excluant l'hypothèse de pouvoir se doter d'un équipement propre, il semblait essentiel de basculer dans une logique d'offre largement diffusable, s'exprimant par une forme très tangible, qui concrétise la présence de l'Inrap dans un langage muséographique spécifique. Il fallait donc imaginer des expositions de grande qualité visuelle, reconnaissables, dont on assurerait entièrement la production. Mais aussi des expositions innovantes - dans la forme et dans le discours - pour que le contenant et le contenu affirment de concert l'engagement de l'institut auprès des publics. Mais quel engagement au juste?

Montage d'une Archéocapsule.

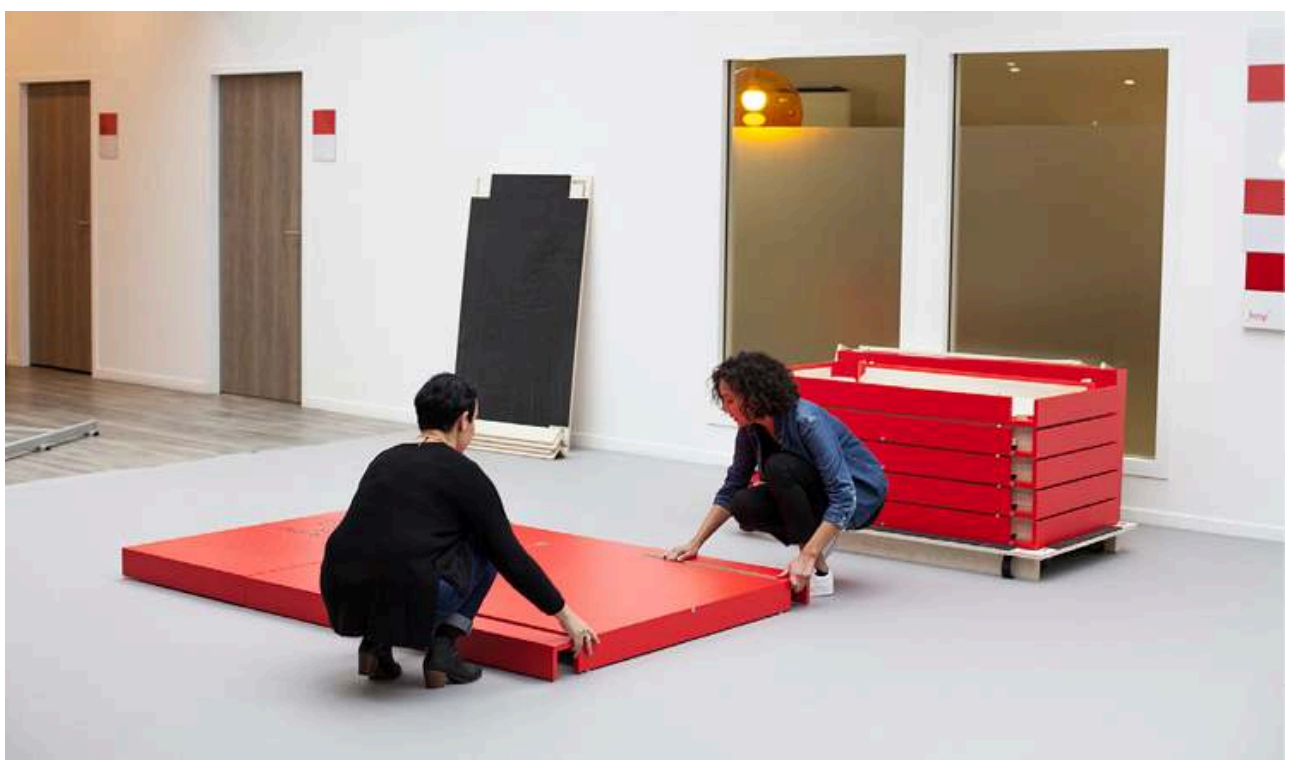



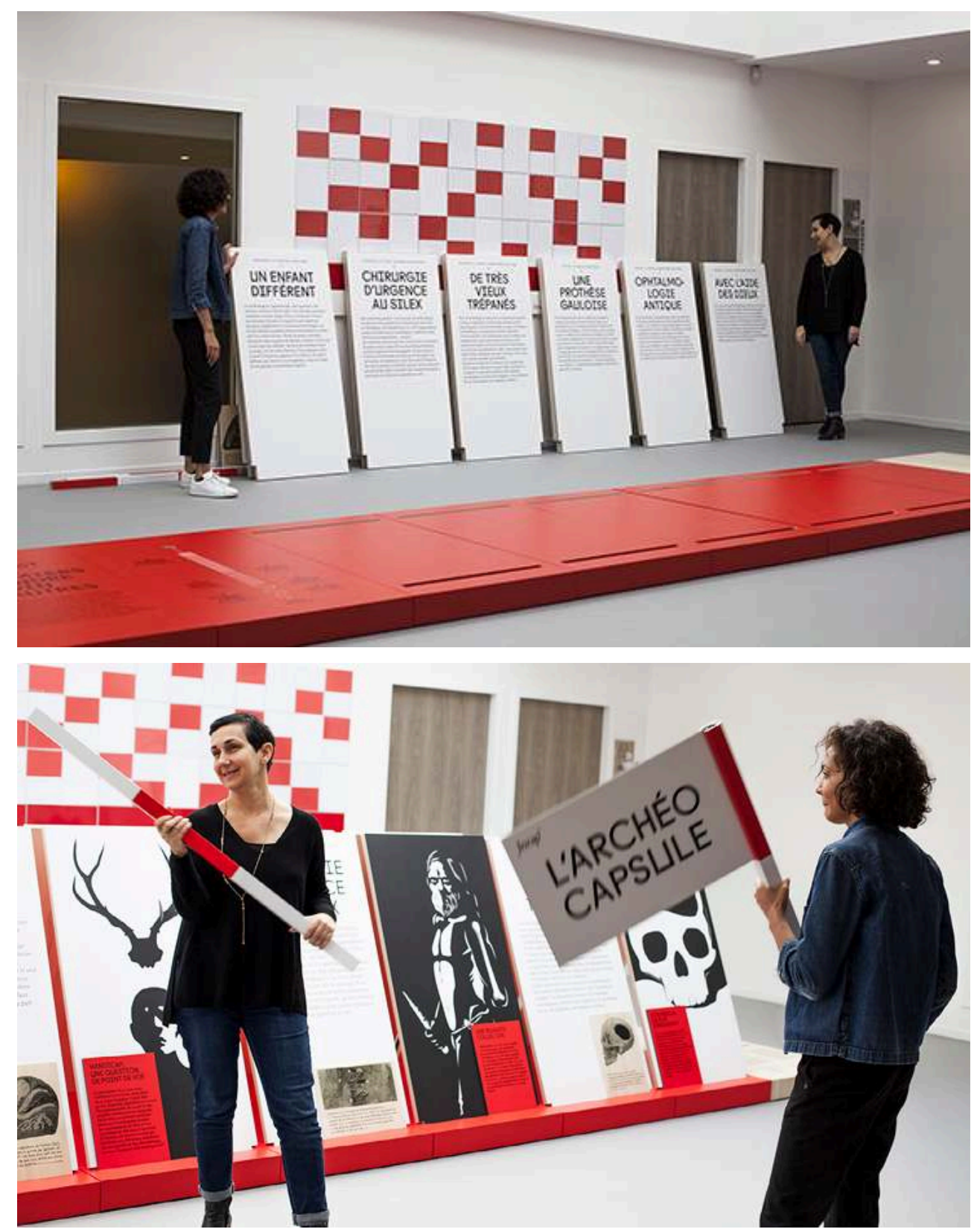

(c) Thanh Phong Lê/Travaux Pratiques

\section{Assumer un discours}

7 À quoi sert l'archéologie? Telle est la question. Entre les connaissances essentielles qu'elle apporte à notre construction du passé et la fascination naturelle qu'elle exerce sur le public, l'archéologie ne semble pas avoir besoin de justification. Pourtant, nous vivons dans une époque plus que jamais à la recherche de sens. L'archéologie participe des disciplines le plus à même d'en pourvoir, pour peu que l'on fasse un pas de côté et que l'on sorte aussi bien de la démonstration scientifique per se, que du discours artistique qui recoupe l'histoire de l'art; mais aussi que l'on reconsidère sa portée humaine et sociétale. Car l'archéologie a ceci d'incomparable qu'elle parle à tout un chacun avec des objets du quotidien d'hommes et femmes venus de passés lointains. En 
inscrivant notre temps et nos préoccupations dans le fil d'une humanité multimillénaire, l'archéologie s'adresse au plus grand nombre. Le télescopage temporel est vertigineux, à mesure de la reconnaissance de soi et de ses propres questionnements.

Ces liens humains et politiques au sens large, que l'archéologie permet et que nombre d'éminents archéologues s'attachent à souligner, peuvent être d'autant plus lisibles si les sujets sont abordés sous un angle original, faisant écho aux questions d'actualité. Aussi, il a semblé fondamental que ces nouvelles productions s'insèrent dans le droit fil des problématiques abordées par les colloques annuels de l'Inrap - violence, migrations, santé, esclavage... ${ }^{3}$ - et qu'elles portent à leur tour un discours foncièrement humain et sociétal, diachronique et universel.

9 L'étendue des fouilles préventives effectuées depuis 30 ans en France - dessinant une carte archéologique vaste et fine du territoire - permet et légitime qu'à côté du gigantesque corpus de données et de recherches archéologiques, des approches globales, transversales et porteuses d'un autre discours, puissent voir le jour pour le public.

\section{Définir un produit culturel}

10 Avec ces paramètres en tête, et à la suite d'une période exploratoire conduite à l'aide d'une mission d'assistance à maîtrise d'ouvrage confiée à l'agence Bureau Oblique, il a été possible de définir les caractéristiques du " produit».

11 D'abord, il fallait que celui-ci respecte le rôle « d'hyper-lieu-ressource » que l'Inrap a réussi à se constituer, sans le dénaturer. Il devait donc entrer dans le corpus d'outils d'interprétation et de valorisation de l'archéologie, tel que depuis dix ans l'Inrap en crée notamment par des publications papier, audiovisuelles et multimédia. Il devait aussi rester ouvert et en quelque sorte modeste sur les utilisations possibles, car tous ces outils allaient alimenter des politiques culturelles et des actions locales, par le biais d'accords de partenariat avec l'Inrap.

Enfin il fallait que ce nouveau produit défende un vrai discours et qu'il puisse poser un regard inédit sur l'archéologie en lui donnant du sens pour les visiteurs d'aujourd'hui.

On peut ainsi résumer le cahier des charges :

- un outil de diffusion « hors les murs» dans le cadre spécifique d'une institution «sans murs »;

15 - posséder une très grande qualité/une matérialité muséale ;

16 - s'afficher clairement comme une production Inrap ;

17 - être en capacité de traiter des thématiques contemporaines, définies parallèlement par un comité scientifique ;

18 - pouvoir servir une logique fortement partenariale, en permettant des adaptations/ augmentations en contenu et en configuration, selon les cas ;

19 - comporter des coûts de fabrication relativement faibles ;

20 - être facile à monter, démonter, stocker et transporter. 
Le début de l'exposition présente l'angle de vue de l'archéologie sur le sujet et situe chronologiquement les différents exemples de recherche.

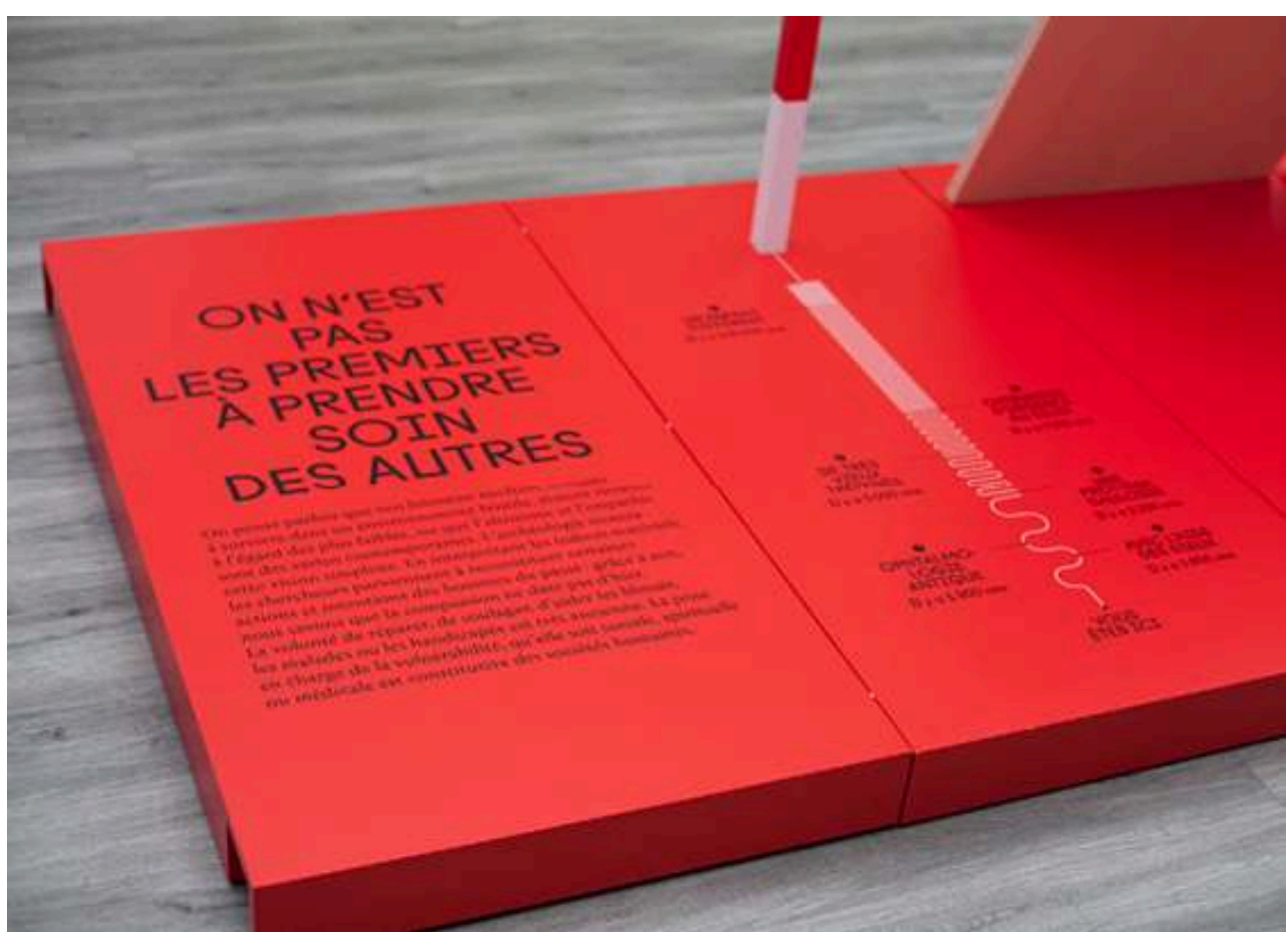

(c) Inrap

Un design pensé pour faciliter le stockage et le transport de l'exposition une fois démontée.

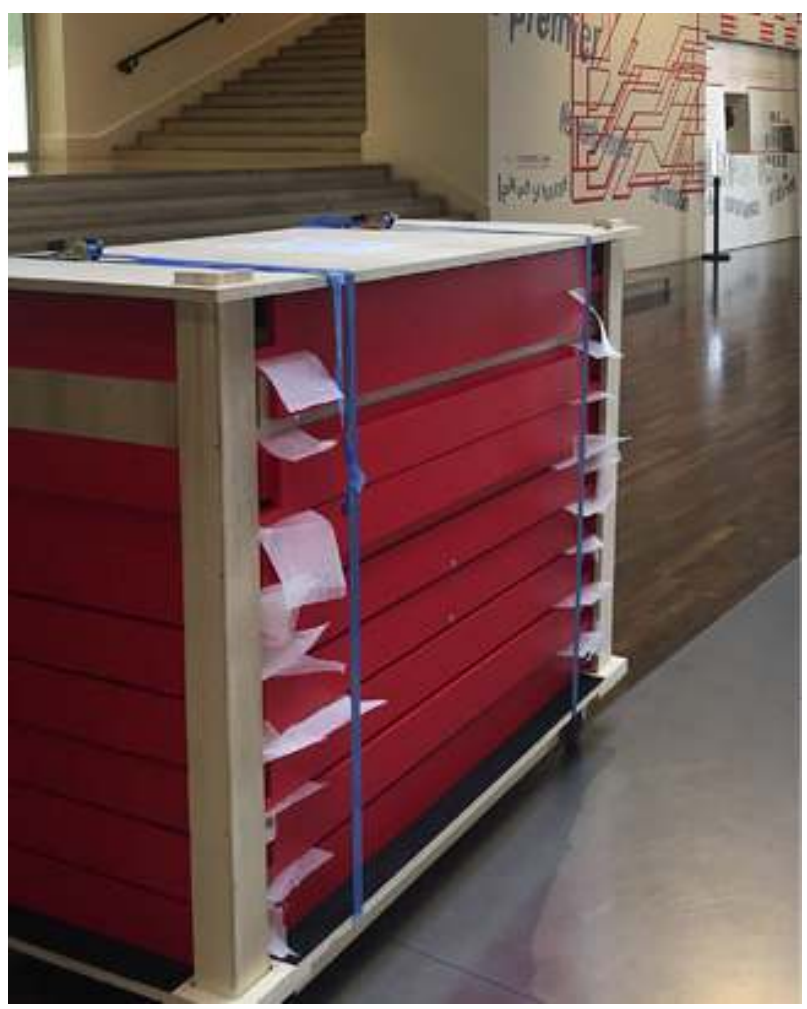

(c) Inrap 
Parallèlement et conjointement à la construction du cahier des charges - avec une modalité non cloisonnée visant à implémenter réciproquement les contenus et la forme - le travail, avec un comité scientifique, s'est axé sur la définition des thématiques, en sélectionnant les sujets contemporains pour lesquels l'archéologie peut apporter un éclairage, un questionnement, un point de vue inattendu. Ainsi ont été retenus les thèmes de Santé, Migrations, Esclavage, Aménagement, Alimentation, Élites et pouvoirs, Le monde des morts, Climats et paysages. Il a été décidé qu'il y aurait pour chaque nouvelle "Archéocapsule» un ou des référents scientifiques dédiés, afin de multiplier les approches et les regards, tout en gardant des choix éditoriaux forts. L'appel d'offres a été remporté par le groupement Designers Unit/Travaux pratiques/Bureau Oblique et ainsi sont nées les Archéocapsules, une flottille d'expositions itinérantes, compactables, modulables, où l'on éclaire par l'archéologie des thématiques d'actualité.

\section{Une présence forte}

Le concept de l'Archéocapsule répond parfaitement et avec une simplicité presque désarmante, à l'ensemble des contraintes et des objectifs posés. Et ceci, tant sur le fond que sur la forme. Objet foncièrement contemporain, elle se compose d'une série de socles sur lesquels viennent s'insérer à la verticale par simple glissement, des panneaux ouverts comme les pages d'un livre.

Texte et image s'appréhendent en lecture simultanée : six exemples archéologiques, six illustrations par des artistes, et six questions contemporaines se présentant, pour filer la métaphore éditoriale, comme des encadrés. Le tout est précédé par un panneau générique qui situe la problématique contemporaine dans l'angle de vue de l'archéologie, et d'un panneau qui situe les exemples archéologiques sur une échelle chronologique. Démontée, elle se présente comme un cube sanglé et arrimé par un cadre en bois à une plateforme à roulette.

24 L'impact visuel est fort. L'objet se pose au sol avec une présence affirmée, mais accueillante, ses proportions sont maîtrisées et la qualité du matériau - un contreplaqué en peuplier façonné en panneaux évidés - exerce un attrait pour les sens.

D'une part, le choix des couleurs, avec un rouge très vif pour les socles, un drapeausignal soutenu par une mire blanc-rouge en guise de mât, un fond blanc pour les panneaux, ancre littéralement dans le sol l'identité visuelle de l'Inrap (qui a fait depuis 2005 du blanc, noir et rouge ses couleurs institutionnelles) tout en étant rapidement identifiable au sein des lieux d'accueil.

D'autre part, les illustrations très grands formats (confiées pour chaque thématique à un artiste différent) donnent à chaque Archéocapsule son identité propre et génèrent un effet de série ou de "collection». Plus encore, ces illustrations proposent une première interprétation, inattendue et imaginative à chaque thématique. C'est un signal fort pour le visiteur, une autorisation à penser l'archéologie autrement, et surtout à réfléchir et à ressentir par lui-même.

\section{Un outil appropriable}

On en vient donc à ce qui est à notre sens le plus intéressant dans ce projet, à savoir les appropriations possibles. L'Archéocapsule lance un sujet sans s'imposer, ni 
physiquement, ni dans le discours. Au public de relever, enrichir, continuer les réflexions qu'elle propose.

Du point de vue du contenu, elle ne vise évidemment pas l'exhaustivité, ni le cumul de notions. Un langage simple mais subtil et engageant est employé. Des gabarits volontairement contraints à 700 signes pour les panneaux, et 300 pour les questions, encouragent la lecture. Le propos est fort, mais concis et surtout ouvert, se terminant par une question ou une réflexion. Face à une trépanation néolithique, à une prothèse gauloise, à un ex-voto médiéval, que peut-on dire de la prise en charge, de l'entre-aide, de l'organisation des soins de nos sociétés actuelles? Face aux migrations des premiers Sapiens depuis l'Afrique, aux déplacements de populations dans l'Empire romain, aux « invasions barbares » du Moyen-Âge, peut-on réinterroger l'actualité des migrations?

L'Archéocapsule laisse à l'institution accueillante, ainsi qu'aux visiteurs, la possibilité d'apporter leurs propres réponses. On aime à les considérer comme un « déclencheur de la pensée ", qui non seulement souhaite mais aussi nécessite la participation de ses usagers et utilisateurs. La possibilité offerte aux partenaires d'adjoindre des panneaux ad hoc, selon leurs souhaits et opportunités (fouilles menées sur le territoire, objets de collection), les multiples configurations envisageables dans l'espace, la possibilité de l'utiliser comme point de départ pour une visite, une exposition, une conférence, un débat..., viennent renforcer cette forme de modestie généreuse.

C'est précisément dans la dialectique entre positionnement fort et utilisation souple, entre discours clair et assumé et dialogue ouvert, que l'Archéocapsule trouve, nous semble-t-il, ses plus grandes raisons d'intérêt.

\section{L'Archéocapsule Migrations à la Maison des Métallos}

L'Archéocapsule Archéologie des Migrations questionne le thème des migrations en les resituant dans le temps très long et en soulignant la pulsion universelle aux mouvements que l'archéologie affirme depuis la sortie d'Afrique de l'Homme, il y a 2 millions d'années. La Maison des Métallos à Paris a été la première institution à l'accueillir, en décembre 2018. Sous la direction de Philippe Mourrat et Catherine Rigeade, ce lieu était bien connu pour ses engagements vis-à-vis du public. Le lieu s'est avéré d'autant plus pertinent, que le festival Migranscène piloté par la Cimade allait être accueilli dans ses murs. Exposer l'Archéocapsule sur ce temps événementiel, envisager l'apport de l'archéologie sur la question des migrations prenait alors tout son sens. 
Dans le cadre d'accueil de groupe, l'Archéocapsule peut être utilisée comme support au débat spontané ou pour expérimenter une médiation à double regard, comme ici, à la Maison des Métallos.
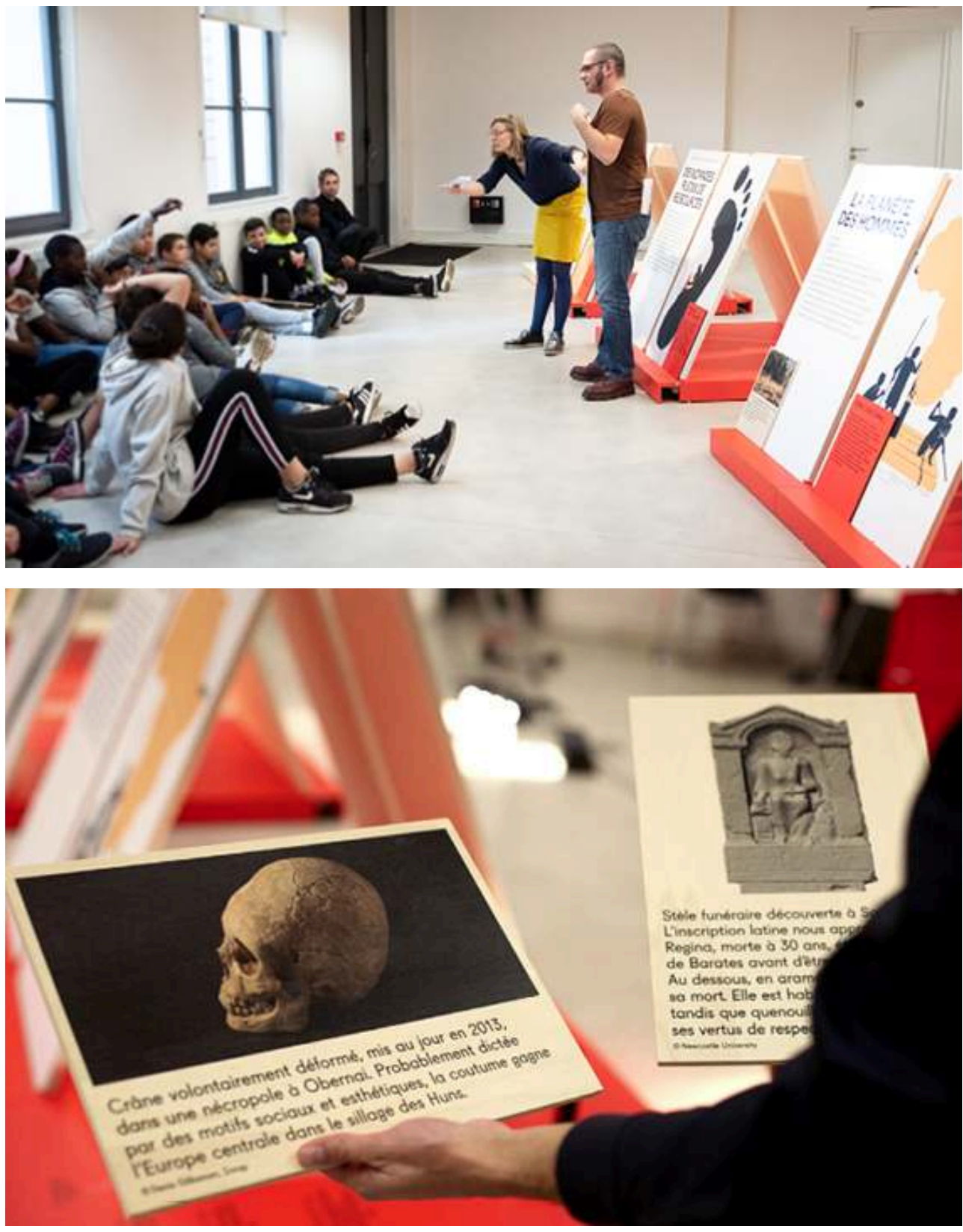


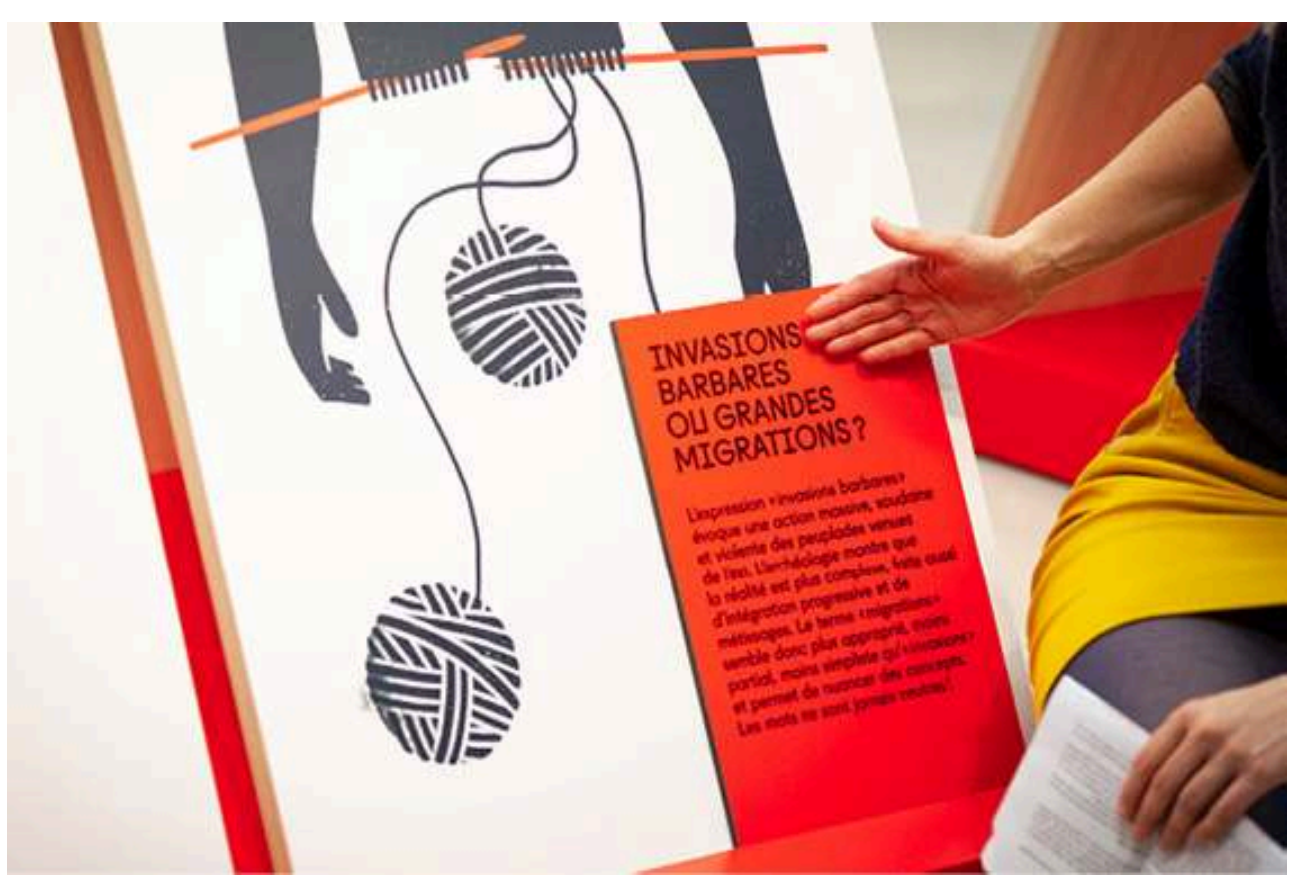

(c) H. Azmoun/Inrap

Réalisée sous la direction scientifique de Dominique Garcia, président de l'Inrap, et illustrée brillamment par le canadien Sébastien Thibauld, l'Archéocapsule a bénéficié d'une animation conjointe de l'Inrap et de la Cimade en direction des publics de la Maison des Métallos.

Un archéologue de l'Inrap et un médiateur de la Maison des Métallos ont accueilli sur une semaine l'ensemble des classes et groupes divers du réseau associatif et du champ social. Dans ce cas de figure, l'Archéocapsule a été utilisée comme support pour provoquer un débat spontané, et pour expérimenter une médiation à double regard, à laquelle s'ajoutait la voix du public.

Cette initiative a nécessité l'implication de trois partenaires et la construction de canevas de visite concertée en amont entre les médiateurs, dans ses grandes lignes, pour susciter et encourager réactions et débats auprès des visiteurs. À cette occasion des conférences mettant en confrontation directe archéologues, acteurs institutionnels et historiens ont également été programmées.

\section{L'Archéocapsule Esclavage au musée de l'Homme}

L'Archéocapsule Archéologie de l'esclavage, sous la responsabilité scientifique de l'archéologue-anthropologue Thomas Romon (Inrap), aborde le sujet sensible de la traite, pour laquelle l'archéologie propose des témoignages matériels particulièrement prégnants. Comme on peut le lire dans le texte d'introduction, "l'archéologie redonne une voix à ceux qui en sont privés dans les archives écrites, opposant aux oublis volontaires et involontaires une incontestable matérialité ». Soulignant le tournant de la contribution de l'archéologie aux connaissances sur cette période, l'Archéocapsule marque la légitimité voire la nécessité de la discipline à s'exprimer sur le sujet, eu égard au corpus vaste et incontestable des données. Depuis les cachettes en Afrique pour échapper aux razzias, aux épaves de navires bourrées d'entraves et de biens d'échange, aux abris des 
Marrons, aux cimetières apparaissant par érosion sur des plages tropicales ou sous les gratte-ciels new-yorkais, les visiteurs parcourent des exemples concrets, illustrés avec délicatesse par Amélie Fontaine. Les questions qu'on y associe résonnent avec l'actualité : comment peut-on considérer un être humain comme une marchandise? Pourquoi les lois économiques sont toujours les plus fortes?

Autour d'un même sujet, l'Archéocapsule interroge le public sur six questions contemporaines - ici l'esclavage - chacune illustrée par un visuel fort et un exemple archéologique.

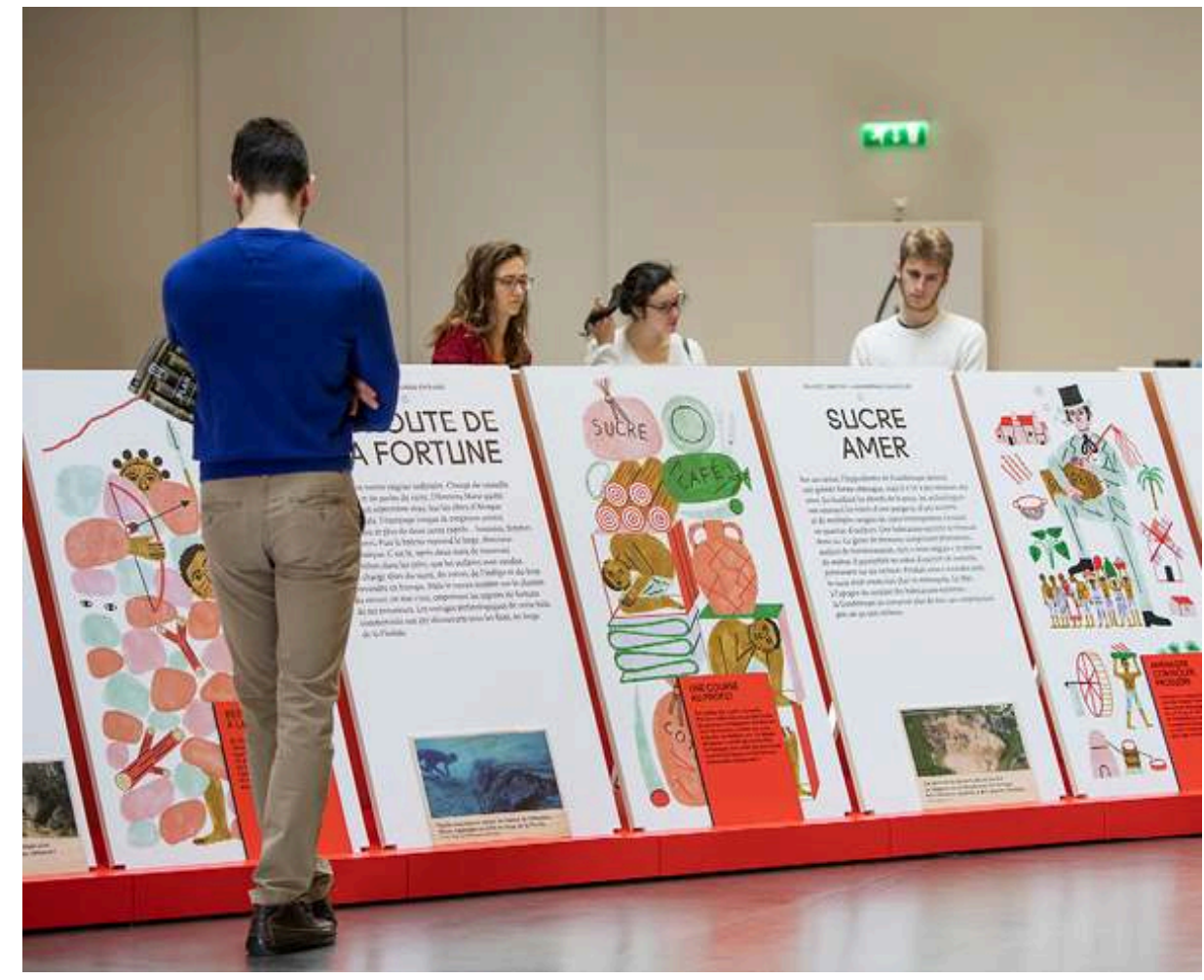

(C) J. Lelièvre/Inrap

Exposée au musée de l'Homme en mai 2019, à l'occasion des commémorations pour l'abolition de l'esclavage, l'Archéocapsule a répondu dans ce cas à un projet événementiel, marquant l'un des temps forts d'une saison En Droits dans laquelle l'Inrap était également présent avec l'exposition Tromelin ${ }^{4}$. Une archéologue de l'Inrap accueillait à cette occasion les visiteurs, engageant des conversations libres et spontanées autour de l'Archéocapsule.

\section{L'Archéocapsule Santé au Chronographe de Rezé}

Enfin, on cite le cas exemplaire de l'Archéocapsule Archéologie du Soin et de la Santé, réalisée sous la direction scientifique de l'archéologue-anthropologue Valérie Delattre (Inrap) et enrichie par les illustrations poignantes et oniriques d'Antonin Bertrand/ Travaux pratiques.

Largement issue du colloque éponyme, elle propose en six exemples le thème du soin et de la prise en charge, montrant que "nous ne sommes pas les premiers à prendre soin des autres». Des questions d'empathie, de croyance, d'organisation sociale et de progrès technique y sont évoquées. Elles font réfléchir aux compétences inouïes des hommes 
dès la préhistoire, à la prise en charge contemporaine des individus en besoin et à l'universalité du sentiment de solidarité qui anime les hommes.

L'exposition Prenez Soin de vous du Chronographe de Rézé s'est appuyée sur l'Archéocapsule Archéologie du soin et de la Santé pour amplifier et enrichir le propos initial.

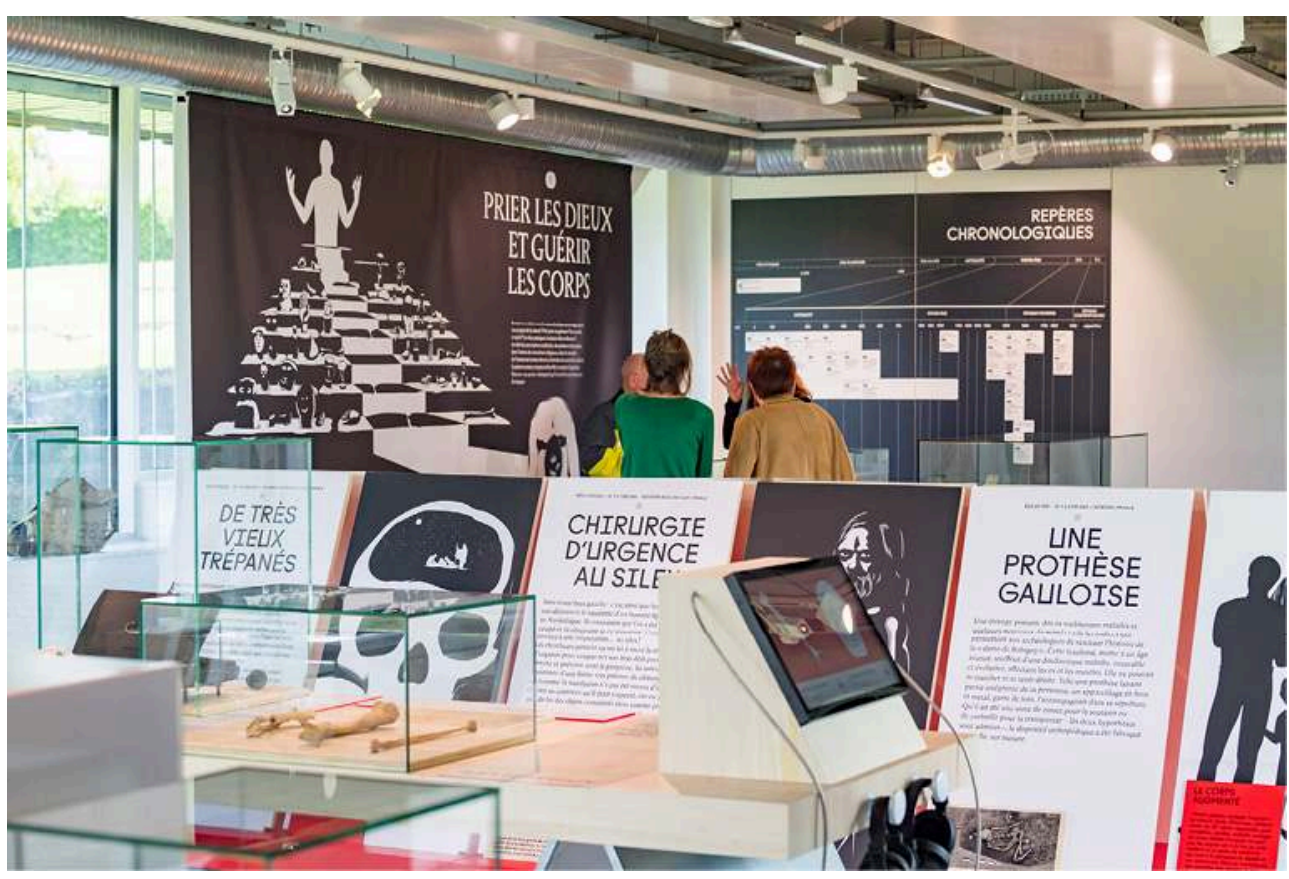

(c) S. Menoret/Nantes Métropole construisant autour de l'Archéocapsule une exposition entière, Prenez soin de vous ${ }^{5}$. La très forte signature visuelle de cette Archéocapsule a investi la totalité du lieu. Son design minimaliste et modulable s'est répercuté sur les supports et vitrines de l'exposition. Son graphisme et ses illustrations ont été créés dans son sillage, l'écriture directe et engageante de ses panneaux a donné le ton de tous les supports écrits. Dans cette scénographie totale, cohérente, sorte d'extension et d'amplification du propos initial, ont trouvé place des objets issus de fouilles archéologiques régionales - crânes néolithiques trépanés, coffrets d'oculistes gallo-romains, biberons et ex-voto antiques, prothèse dentaire Renaissance et plateaux-repas d'hôpitaux modernes - témoins réels des questions posées par l'Archéocapsule.

Une action culturelle intense est organisée autour de l'exposition, comprenant des rencontres régulières avec les archéologues et les médiateurs.

\section{Prospectives}

41 Les Archéocapsules sont, dans une certaine mesure, l'expression d'un pari. $\mathrm{Ne}$ répondant pas à une demande - ou alors à une demande non exprimée - elles incarnent une logique d'offre, construite sur une appréhension de besoins et objectifs propres à l'Inrap, mais aussi sur un élan explorateur et prospectif. Dès lors, ce sont la plus large diffusion possible et le plus grand nombre d'initiatives en direction des publics qui seront les seuls indicateurs de sa réussite. Restent à poursuivre une identification fine des institutions d'accueil potentielles, ainsi qu'un renforcement des modalités de 
communication et démarchage auprès des partenaires. Seule l'expérience permettra sur le plus long terme de tirer des conclusions, des bilans et le cas échéant des réorientations stratégiques et conceptuelles.

\section{NOTES}

1. Groupe de recherche en archéologie navale.

2. "L'archéologie préventive a pour objet d'assurer, à terre et sous les eaux, dans les délais appropriés, la détection, la conservation ou la sauvegarde par l'étude scientifique des éléments du patrimoine archéologique affectés ou susceptibles d'être affectés par les travaux publics ou privés concourant à l'aménagement. Elle a également pour objet l'interprétation et la diffusion des résultats obtenus ", présentation de l'archéologie préventive sur le site Internet du ministère de la culture : www.culture.gouv.fr

3. Inrap, colloques : www.inrap.fr/les-colloques-de-l-inrap-12337

4. Le musée de l'Homme a été la dernière étape de l'exposition itinérante Tromelin, lîle aux esclaves oubliés citée plus haut, entre février et juin 2019.

5. Prenez Soin de vous. Archéologie du soin et de la Santé était présentée au Chronographe de Rezé du 15 juin 2019 au 5 janvier 2020.

\section{RÉSUMÉS}

Dans le cadre de sa mission ressource et afin de s'ouvrir davantage au public, l'Inrap a mis en place un dispositif d'exposition itinérante. Une opportunité, pour les institutions accueillantes, de s'approprier les résultats de recherches récentes et locales, et de donner à voir l'apport de l'archéologie sur des questions de société.

\section{INDEX}

Mots-clés : exposition itinérante ; patrimoine et/ou collection archéologiques ; musée d'histoire et d'archéologie; monument et site archéologique

\section{AUTEUR}

\section{ALESSIA BONANNINI}

Chargée des expositions à l'Inrap

alessia.bonannini@inrap.fr 